Investigation of the impact of transient heat loads applied by laser irradiation on ITER-grade tungsten

This content has been downloaded from IOPscience. Please scroll down to see the full text. 2014 Phys. Scr. 2014014005

(http://iopscience.iop.org/1402-4896/2014/T159/014005)

View the table of contents for this issue, or go to the journal homepage for more

Download details:

IP Address: 134.94.122.17

This content was downloaded on 14/07/2014 at 09:03

Please note that terms and conditions apply. 


\title{
Investigation of the impact of transient heat loads applied by laser irradiation on ITER-grade tungsten
}

\author{
A Huber ${ }^{1}$, A Arakcheev ${ }^{2}$, G Sergienko ${ }^{1}$, I Steudel ${ }^{1}$, M Wirtz $^{1}$, \\ A V Burdakov ${ }^{2}$, J W Coenen ${ }^{1}$, A Kreter $^{1}$, J Linke $^{1}$, Ph Mertens ${ }^{1}$, \\ V Philipps ${ }^{1}$, G Pintsuk ${ }^{1}$, M Reinhart ${ }^{1}$, U Samm ${ }^{1}$, A Shoshin ${ }^{2}$, B Schweer ${ }^{1}$, \\ B Unterberg ${ }^{1}$ and $M$ Zlobinski $^{1}$
}

${ }^{1}$ Institute of Energy and Climate Research, Forschungszentrum Jülich, EURATOM Association, Trilateral Euregio Cluster, D-52425 Jülich, Germany

${ }^{2}$ Budker Institute of Nuclear Physics (BINP), Novosibirsk 630090, Russia

E-mail: A.Huber@fz-juelich.de

Received 14 May 2013

Accepted for publication 11 July 2013

Published 1 April 2014

\begin{abstract}
Cracking thresholds and crack patterns in tungsten targets after repetitive ITER-like edge localized mode (ELM) pulses have been studied in recent simulation experiments by laser irradiation. The tungsten specimens were tested under selected conditions to quantify the thermal shock response. A Nd:YAG laser capable of delivering up to $32 \mathrm{~J}$ of energy per pulse with a duration of $1 \mathrm{~ms}$ at the fundamental wavelength $\lambda=1064 \mathrm{~nm}$ has been used to irradiate ITER-grade tungsten samples with repetitive heat loads. The laser exposures were performed for targets at room temperature (RT) as well as for targets preheated to $400^{\circ} \mathrm{C}$ to measure the effects of the ELM-like loading conditions on the formation and development of cracks. The magnitude of the heat loads was $0.19,0.38,0.76$ and $0.90 \mathrm{MJ} \mathrm{m}^{-2}$ (below the melting threshold) with a pulse duration of $1 \mathrm{~ms}$. The tungsten surface was analysed after 100 and 1000 laser pulses to investigate the influence of material modification by plasma exposures on the cracking threshold. The observed damage threshold for ITER-grade W lies between 0.38 and $0.76 \mathrm{GW} \mathrm{m}^{-2}$. Continued cycling up to 1000 pulses at RT results in enhanced erosion of crack edges and crack edge melting. At the base temperature of $400{ }^{\circ} \mathrm{C}$, the formation of cracks is suppressed.
\end{abstract}

Keywords: divertor material, tungsten, ELM, laser, thermal shock

(Some figures may appear in colour only in the online journal)

\section{Introduction}

A critical issue for the operation of ITER are large power loads to the divertor during intense transient events, such as bursts of edge localized modes (ELMs), which can seriously limit the lifetime of the target plates. Energy densities up to $10 \mathrm{MJ} \mathrm{m}^{-2}$ are predicted for unmitigated type I ELMs [1] in the baseline scenario of ITER operation. To prevent unacceptable erosion, cracks, melting and other damage to the divertor targets, the energy loss per single ELM should be restricted to $\Delta W_{\mathrm{ELM}} \sim$ $1 \mathrm{MJ}$, which corresponds to $\sim 3 \%$ of the plasma stored energy, and to an energy density load of about $0.5 \mathrm{MJ} \mathrm{m}^{-2}$.
For improved lifetime analyses, the simulation of heat and particle loads of such ELMs in laboratory devices is mandatory to determine their impact on plasma-facing materials and the underlying physical processes. Several techniques, such as electron beam irradiation, like in the JUDITH facility [2] and plasma guns such as QSPA [3] and GOL-3 [4] are currently in use to investigate the behaviour of materials under ITER-relevant transient heat loads. Alongside these techniques, high energy lasers can be used to simulate the effect of ELM-like heat loads under laboratory conditions. Papers [5-7] give an overview of different simulation methods including the laser irradiations for transient events and 


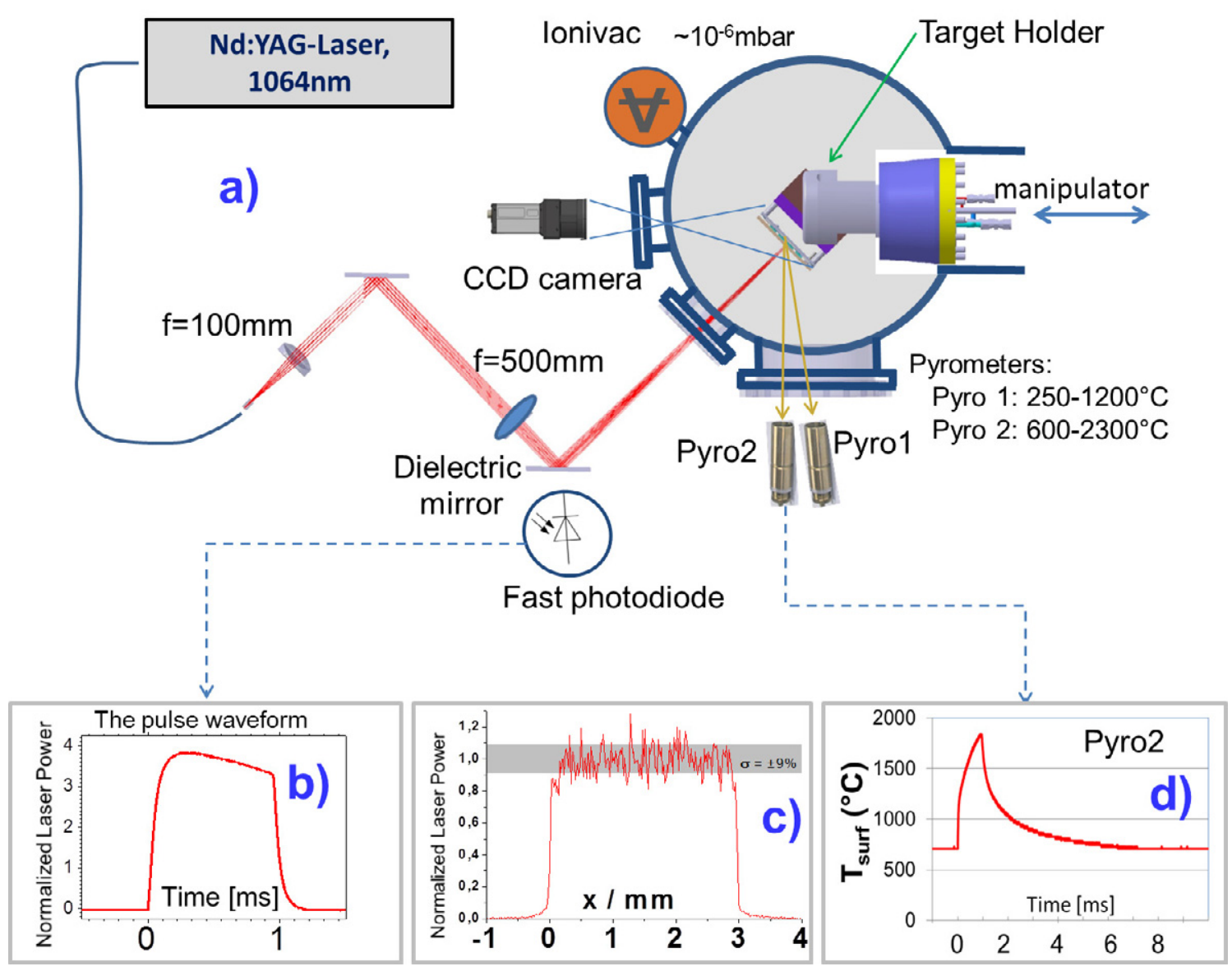

Figure 1. Schema of the laser irradiation setup with laser pulse waveform, spatial distribution and surface temperature evolution during the laser pulse.

damage induced in tungsten. Despite the difference in the penetration depth of the particles used for applying the heat flux in the different facilities, it has been reported that the different techniques show, in general, similar damage behaviours and the same damage thresholds.

Here, a Nd:YAG laser capable of delivering up to $32 \mathrm{~J}$ of energy per pulse at a wavelength of $1064 \mathrm{~nm}$ with a pulse duration of $\tau_{\mathrm{LAS}}=1 \mathrm{~ms}$ has been used for thermal shock studies. With a laser spot size of about $3.1 \mathrm{~mm}$ (spot area around $7.5 \mathrm{~mm}^{2}$ ) and repetition rates of 0.5 and $10 \mathrm{~Hz}$ we would get energy densities absorbed on the loaded surfaces of up to $1 \mathrm{MJ} \mathrm{m}^{-2}$. The present laser system can thus simulate transient heat loads with ITER-like energy densities.

Investigation of the influence of the power density on the $\mathrm{W}$ material modifications in the range from 0.19 to $0.90 \mathrm{GW} \mathrm{m}^{-2}$ at $\tau_{\mathrm{LAS}}=1 \mathrm{~ms}$ will be presented. Additionally, the impact of the frequency of the transient heat loads in the range $0.5-10 \mathrm{~Hz}$ and of the number of cycles (100 and 1000 pulses) on the tungsten surface at different base temperatures will be discussed.

\section{Experimental set-up}

The experimental set-up for ELM simulation experiments using laser irradiation of the investigated targets at Forschungszentrum Jülich (FZJ) is shown in figure 1. A Nd:YAG laser capable of delivering up to $32 \mathrm{~J}$ of energy per pulse with a duration of $1 \mathrm{~ms}$ was used to irradiate tungsten samples with repetitive heat loads (see figure 1(a)). The laser was operated in the free generation mode (without Q-switch). The irradiation area was limited to a small spot of $\sim 3.1 \mathrm{~mm}$ diameter and the power deposition profile in the irradiated area was nearly uniform (see figure 1(c)) to avoid the instrumental influence on the cracking behaviour. The beam of the fibre-coupled Nd:YAG laser at the fundamental wavelength $(\lambda=1064 \mathrm{~nm})$ was introduced through a window in the vacuum chamber nearly perpendicularly to the target surface and imaged by two lenses on the spot to be loaded at the sample surface. To keep the optical losses as small as possible, only dielectric mirrors were used in the optical arrangement. The steering of the location of the laser spot was done by adjustment of the last mirror just in front of the vacuum window. The vacuum vessel was pumped down to $4 \times 10^{-6}$ mbar by a turbo-molecular pump. The target sample was mounted on a movable, rotatable and reclining carrier system that allows the transportation and orientation of the probe inside the analysis chamber. Several tungsten probes of $12 \times 12 \mathrm{~mm}^{2}$ are mounted on top of the target holder, which can be cooled or resistively heated up to $1000{ }^{\circ} \mathrm{C}$. Two one-colour pyrometers with temperature ranges $250-1200$ and $600-2300{ }^{\circ} \mathrm{C}$ monitored the surface temperature of the spot centre during the laser pulse with a time resolution of $10 \mu \mathrm{s}$ (see figure 1(a) with schematic arrangements of the pyrometers and, for the time evolution of the surface temperature, figure 1(d)). Additionally, the target temperature was controlled by a thermocouple. In the 
presented experiments the samples have been exposed to ELM relevant power densities between 0.19 and $0.90 \mathrm{GW} \mathrm{m}^{-2}$. These values take into account the laser absorption rate of $26 \%(T \times(1-R))$ due to losses in the optical path (transmittance of $T=64 \%$ ) and due to reflection of the laser energy at the sample (reflection of $R=60 \%$ ) itself. The pulse waveform was measured by a fast photodiode located behind the last dielectric mirror, as shown in figure 1(b), demonstrating the constant power during the pulse duration as well as the smooth temporal behaviour (no spikes which could lead to material ablation).

The penetration depth of the energy deposition is negligible for the laser $(\approx 17 \mathrm{~nm})$, similar to the case of fusion devices and is always small in comparison to the temperature decay length at the end of the laser pulse.

Samples used in these studies were cut by wire (electrical discharge machining) from tungsten blocks to the dimensions $12 \times 12 \times 5 \mathrm{~mm}^{3}$. The material used was an ITER-grade tungsten, manufactured by Plansee AG [8], with a purity of $99.5 \mathrm{wt} \%$. It was produced by powder metallurgical routes (cold iso-static pressing of homogenized powder for the green stuff, subsequent sintering at $2000-2500{ }^{\circ} \mathrm{C}$ ) and deformed by forging or rolling of the sintered blanks into rod-like geometries. The deformation processes induce preferential grain orientations. The grains of the tungsten specimens were oriented perpendicularly to the loaded surface with a typical grain length around $110 \mu \mathrm{m}$ and width of about $40 \mu \mathrm{m}$ (parallel to the surface). Typically, the cracking behaviour depends on the orientation of the heat loads with respect to the grain orientation.

Before exposure, the surface of all samples was polished to a mirror finish with a roughness $R_{\mathrm{a}}$ of less than $0.1 \mu \mathrm{m}$. The roughness parameter $R_{\mathrm{a}}$ is defined as the arithmetic average of the deviation from the average height. After laser irradiation, the samples were analysed by optical microscopy, scanning electron microscopy (SEM) and laser profilometry.

\section{Result and discussion}

\subsection{Variation of power density}

The base temperature of the $\mathrm{W}$ samples before thermal shock exposure was around room temperature (RT), i.e. below the ductile-to-brittle transition temperature (DBTT) of $\sim 500-700 \mathrm{~K}[9]$. With the repetition rate used for these tests of $0.5 \mathrm{~Hz}$ and with an actively cooled target holder the base temperature of the test samples increased by $80 \mathrm{~K}$ at most. SEM images of ITER-grade tungsten samples exposed each to 100 laser beam pulses in the power density range between 0.19 and $0.90 \mathrm{GW} \mathrm{m}^{-2}$ are shown in figure 2 (top). The ITER-grade $\mathrm{W}$ sample exhibit a damage threshold between 0.38 and $0.76 \mathrm{GW} \mathrm{m}^{-2}$ for 100 heat pulses, which is higher than shown for high purity tungsten in $[6,7]$. The SEM analysis of loaded surface (figure 2 (bottom)) shows the formation of the sub-grain boundaries with size of $\approx 2 \mu \mathrm{m}$ that are much smaller than the original grain sizes. The maximum surface temperature of the samples has been measured at each pulse during the 100 pulses series. It reached about $3000 \mathrm{~K}$ at highest power density and is below the melting point. Due to surface modification
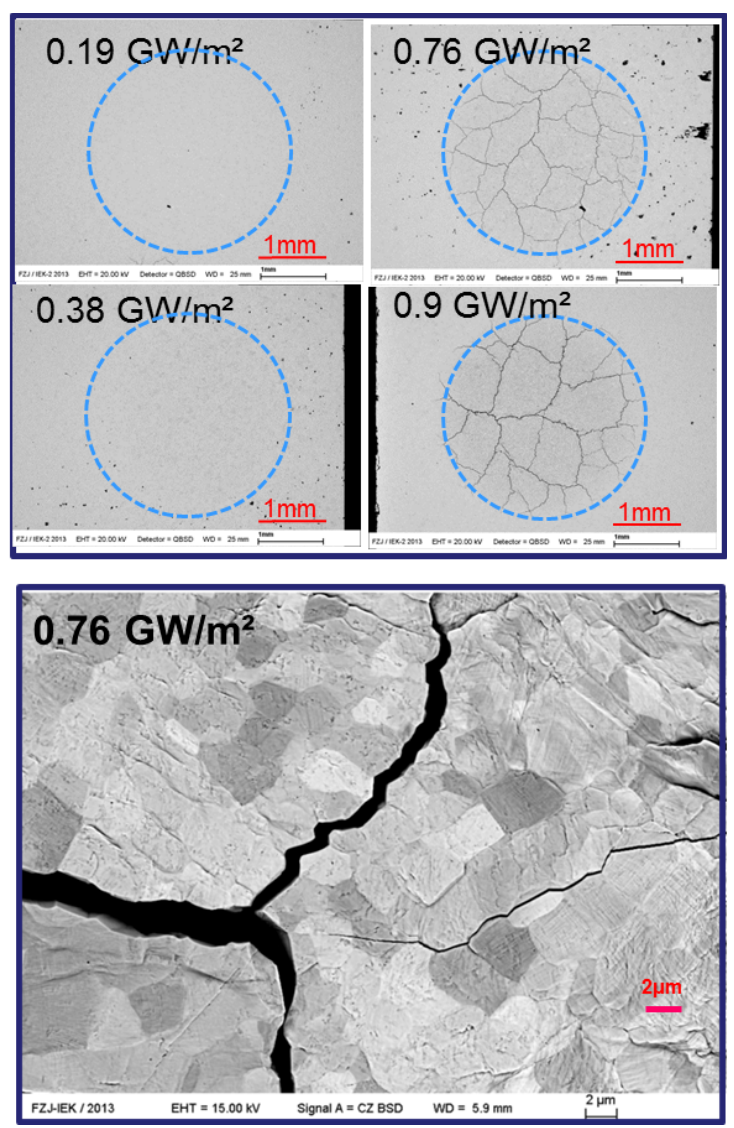

Figure 2. SEM images (top) of the ITER-grade tungsten samples after 100 pulses of laser beam exposure at RT. The circles on the figure indicate the spot sizes of the laser loads on the loaded samples; SEM figure (bottom) with high magnification at absorbed power density of $0.76 \mathrm{GW} \mathrm{m}^{-2}$.

such as roughening, which will be discussed later in this contribution, the reflection of the laser beam on the $\mathrm{W}$ target decreases from 60 to $55 \%$ for polished and exposed $\mathrm{W}$ correspondingly leading to an increase of the absorbed power by $\approx 12.5 \%$.

No melting occurred in the observed power density range up $P=0.90 \mathrm{GW} \mathrm{m}^{-2}$ for 100 laser pulses, while major cracks were formed. These values correspond to heat flux factor values $\left(F_{\mathrm{HF}}=P \times t_{\mathrm{LAS}}^{1 / 2}\right)[10]$ which determines the surface temperature rise caused by transient heat load event, between 6 and $28.5 \mathrm{MW} \mathrm{m}^{-2} \mathrm{~s}^{1 / 2}$.

\subsection{Variation of pulse number and base temperatures}

It has been shown in [11] that the degradation of thermal shock loaded tungsten increased with the number of pulses. In this contribution, the impact of the number of pulses (100 and 1000) on the tungsten surface at different base temperatures has been analysed. To keep the base temperature of the tested samples before each laser exposure pulse below $100^{\circ} \mathrm{C}$ (and, correspondingly, below DBTT), the 1000 pulses experiment was performed in 10 series of 100 pulses each with a sufficiently long dwell time between each series to allow the base temperature to return to RT. Figure 3 shows SEM images of the ITER-grade tungsten after 100 pulses (figure 3(a)) and 1000 pulses (figure 3(b)) of laser beam exposure at RT as well as after 1000 

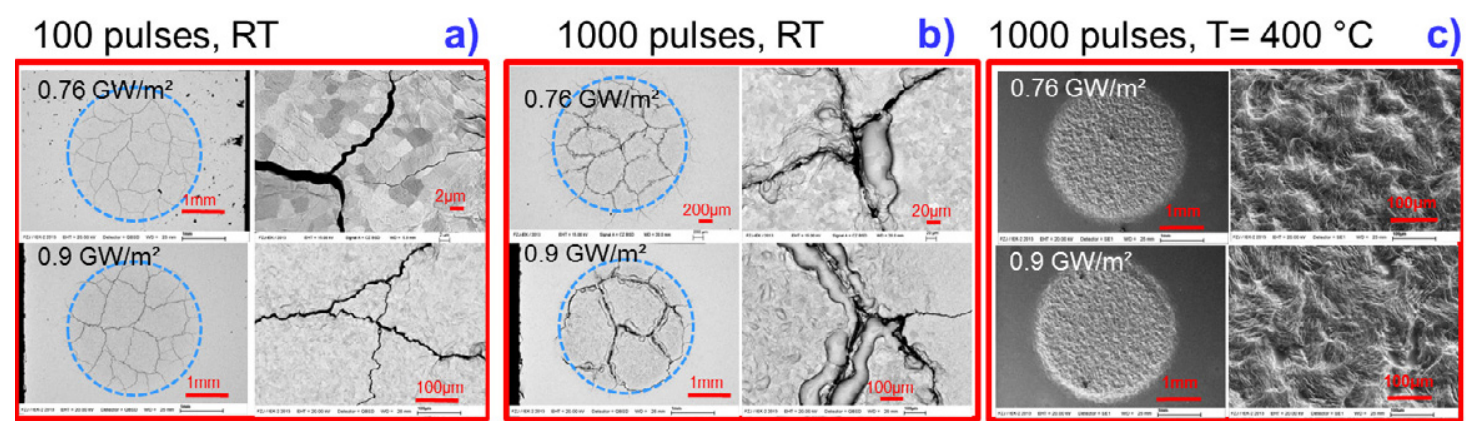

Figure 3. Different damage types observed after the exposure of tungsten to thermal shock events: (a) thermal shock-induced crack networks after 100 heat load pulses, (b) crack formation with enhanced erosion of the crack edges and melting of edges and (c) surface modifications like roughening without crack formation on the tungsten exposed to 1000 laser beam pulses at $T_{\text {surf }}=400^{\circ} \mathrm{C}$.

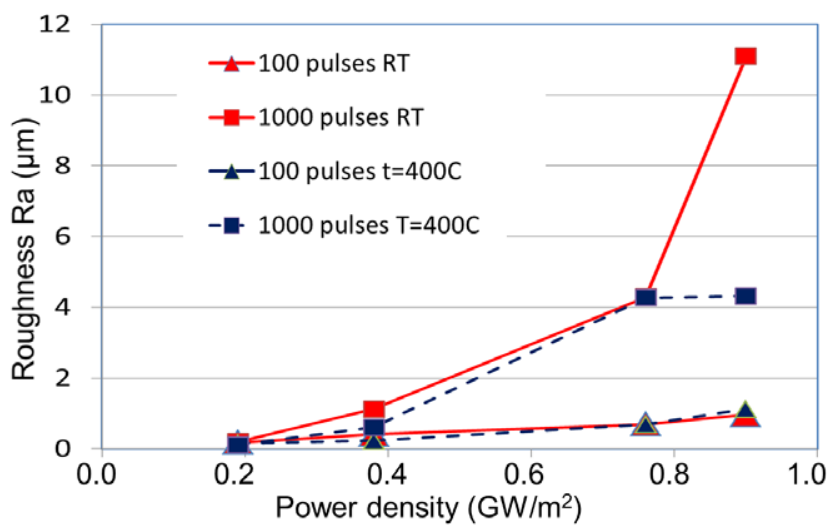

Figure 4. Power density dependence of the arithmetic mean roughness $\left(R_{\mathrm{a}}\right)$ after laser beam exposure.

heat pulses at $400^{\circ} \mathrm{C}$ surface temperature (figure 3(c)). Before exposure, the surface of the samples was polished to a mirror finish with roughness $R_{\mathrm{a}}$ of less than $0.1 \mu \mathrm{m}$. The surface roughness $R_{\mathrm{a}}$ caused by 100 thermal shock loads increased with power density to $1 \mu \mathrm{m}$ at $0.90 \mathrm{GW} \mathrm{m}^{-2}$ as shown in figure 4 and is identical for both investigated base temperatures. This change in surface morphology comes from thermal fatigue effects. In the tackled pulse number range, the average roughness $\left(R_{\mathrm{a}}\right)$ at RT increased almost proportionally to the number of pulses, e.g. 1.0 and $11 \mu \mathrm{m}$ for 100 and 1000 pulses, respectively, at an absorbed power density of $0.90 \mathrm{GW} \mathrm{m}^{-2}$. The significantly increased roughness $R_{\mathrm{a}}$ may be a consequence of a higher number of compressive-tensile cycles. No dependence of the surface roughness $R_{\mathrm{a}}$ on the base temperature of the samples has been observed: $R_{\mathrm{a}}$ for the two base temperatures is almost the same except at $0.9 \mathrm{GW} \mathrm{m}^{-2}$, Despite the fact that the measured temperature was below $3000 \mathrm{~K}$ and correspondingly below the melting point, the SEM analysis of the exposed samples at $400^{\circ} \mathrm{C}$ surface temperature and at $0.9 \mathrm{GW} \mathrm{m}^{-2}$ shows localized melting of the largest peaks on the loaded surface resulting in the saturation of the $R_{\mathrm{a}}$ for power densities beyond $0.76 \mathrm{GW} \mathrm{m}^{-2}$. Thus, this localized melting is responsible for different values of $R_{\mathrm{a}}$ at two base temperatures at $P=0.9 \mathrm{GW} \mathrm{m}^{-2}$.

The observed cracking behaviour is the formation of a network with an average distance between cracks of $470 \mu \mathrm{m}$ for 100 pulses and $390 \mu \mathrm{m}$ for 1000 heat loads. The observed crack width varied between 4 and $10 \mu \mathrm{m}$ at 100 pulses but increased strongly with large pulse numbers, e.g. for 1000 pulses at $0.9 \mathrm{GW} \mathrm{m}^{-2}$ from 5 to $18 \mu \mathrm{m}$.

Whereas no melting has been observed for 100 laser pulses, continued cycling up to 1000 pulses after crack formation results at RT and at power densities of 0.76 and $0.90 \mathrm{GW} \mathrm{m}^{-2}$ in the erosion of crack edges by melting. The enhanced erosion of crack edges as well as crack melting has been observed in previous experiments by electron beam exposure [11]. In this analysis the erosion was explained by mechanical fatigue of crack surfaces and prominent crack edges that repeatedly rubbed against each other at every pulse due to cyclic thermal expansion. This mechanism caused an accumulation of plastic deformation at the crack edges, which also explains the enhanced roughening around edges and the often observed increased height of crack edges, the so-called lifting. Metallographic cross sections after 100 and 1000 pulses at $0.76 \mathrm{GW} \mathrm{m}^{-2}$ have been made across the laser exposure spot (see figure 5). At high pulse numbers the friction at crack edges can cause erosion and high accumulative plastic deformation leading to a reduced heat transfer capability or even thermal isolation of the protruding parts of the W material, which can melt. Molten droplets as well as dust produced by erosion of the crack edges can have serious consequences on the plasma performance when ejected towards the main chamber and penetrating the plasma.

While it takes only just a few pulses to form large brittle cracks, the formation of small cracks is related to thermal fatigue and their growth towards a crack network is a function of the pulse number. The typical cracks occurring in ITER-grade W material body, observed by the cross-section metallography analysis, run along grain boundaries where the strength of the material is lower. No cracks parallel to the loaded surfaces have been observed during the thermal shock loading.

At $400^{\circ} \mathrm{C}$ base temperature the formation of cracks is suppressed. At this temperature the tungsten becomes more ductile and is able to compensate the stresses by plastic deformation without exceeding the fracture strength. The SEM images after 1000 heat pulses at $400^{\circ} \mathrm{C}$ surface temperature (figure 3(c)) confirm this statement. At base temperature of $400^{\circ} \mathrm{C}$ only surface modification such as roughening occurs. The surface roughness $R_{\mathrm{a}}$ at this temperature increases very fast to a maximum value of $\approx 4.0 \mu \mathrm{m}$ at $0.76 \mathrm{GW} \mathrm{m}^{-2}$ and stays constant for higher power densities. 


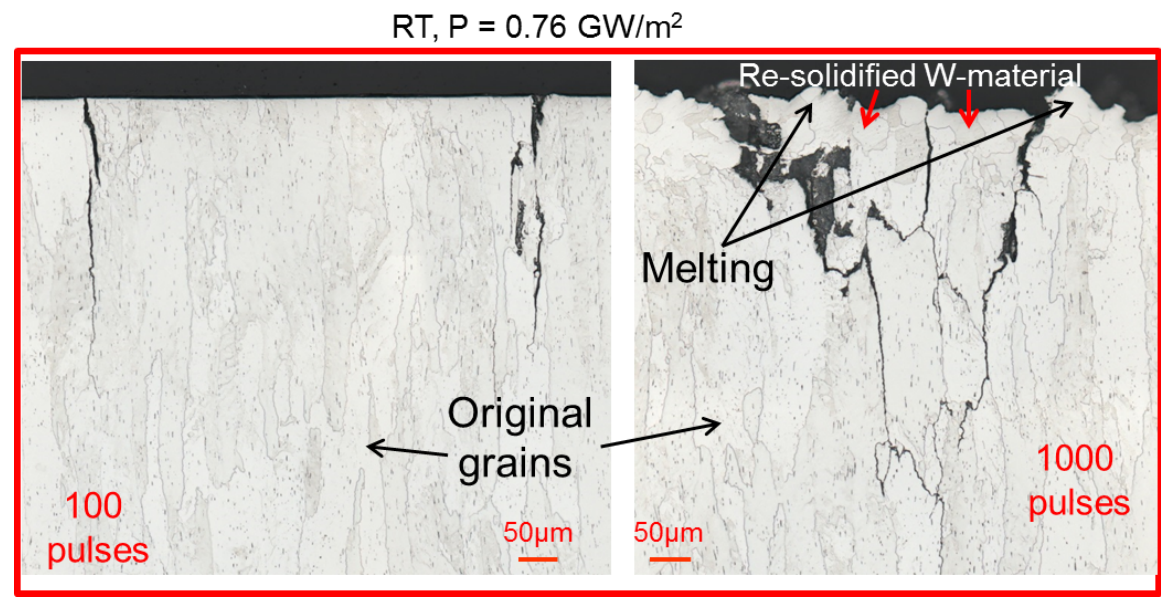

Figure 5. Cross section image of samples loaded with 100 (left) and 1000 (right) pulses of power density $0.76 \mathrm{GW} \mathrm{m}^{-2}$ at $T_{\text {surf }}=20^{\circ} \mathrm{C}$. The original grain structure is shown at higher depths (bottom).

\subsection{The dependence of the surface modification on the frequency of the transient heat loads}

The impact of the frequency of the transient heat loads has been investigated by exposing the $\mathrm{W}$ samples also to 1000 laser pulses at RT with a repetition rate of $10 \mathrm{~Hz}$. As mentioned above for the $0.5 \mathrm{~Hz}$ case, the investigated $\mathrm{W}$ grade shows no observable surface damage for $0.38 \mathrm{GW} \mathrm{m}^{-2}$ and 100 heat pulses. In contrast, at the same loading conditions and a loading frequency of $10 \mathrm{~Hz}$ a crack network was formed. The increased frequency of the thermal shock loads probably leads to an enhanced accumulation of plastic deformation in the region of the loaded surface and, correspondingly, to a reduced damage threshold.

\section{Conclusions}

To examine the performances of ITER-grade $\mathrm{W}$ under thermal shock loads, simulations were performed using the laser beam facility at Forschungszentrum Jülich. For energy densities beyond the damaging threshold, thermal shock crack networks as well as surface roughening are formed on the loaded surface of the investigated ITER-grade W. The observed damage threshold is located between 0.38 and $0.76 \mathrm{GW} \mathrm{m}^{-2}$. The typical cracks occur at grain boundaries where the strength of the material is lower and due to the grain orientation perpendicular to the loaded surface no cracks parallel to the loaded surface were observed as a result of the thermal shock loading. In case of crack formation, continued cycling up to 1000 pulses results in enhanced erosion of crack edges by melting. At high pulse numbers the friction at crack edges can cause erosion and high accumulative plastic deformation leading to a reduced heat transfer capability or even thermal isolation of the protruding parts of the $\mathrm{W}$ material, leading to material melting. No crack formation was found at the base temperature of $400{ }^{\circ} \mathrm{C}$ during the exposure to thermal shock events. At such temperatures tungsten becomes more ductile and is able to compensate the stresses by plastic deformation without exceeding the fracture strength. An increase of the frequencies of the heat cycles to $10 \mathrm{~Hz}$ may effectively decrease the damage threshold, which has to be taken into account when mitigating ELMs in ITER via an increase in frequency.

\section{Acknowledgments}

This work, supported by the European Communities under the contract of Association between EURATOM and FZJ, was carried out within the framework of the European Fusion Development Agreement (EFDA). The views and opinions expressed herein do not necessarily reflect those of the European Commission. This work was financially supported by the Ministry of Education and Science of the Russian Federation. The authors would like to acknowledge the support by B Riccardi and P Lorenzetto from F4E and thank them for providing ITER-grade W samples.

\section{References}

[1] Loarte A et al 2007 Phys. Scr. T128 222

[2] Duwe R, Kuhnlein W and Munstermann H 1995 SOFT 1994: Proc. 18th Symp. on Fusion Technology (SOFT) (Karlsruhe, Aug. 1994) p 355

[3] Garkusha I E, Arkhipov N I, Klimov N S, Makhlaj V A, Safronov V M, Landman I and Tereshin V I 2009 Phys. Scr. T138 014054

[4] Burdakov A et al 2007 Fusion Sci. Technol. 51(2T) 106

[5] Wirtz M et al 2011 Phys. Scr. T145 014058

[6] Wirtz M et al 2013 J. Nucl. Mater. 438 S833

[7] Huber A et al 2013 Fusion Sci. Technol. 63(1T) 197

[8] Plansee Group Homepage 2012 www.plansee.com/de/index.htm

[9] Davis J W et al 1998 J. Nucl. Mater. 258-263 308

[10] Riccardo V et al 2005 Nucl. Fusion 451427

[11] Loewenhoff Th W, Linke J, Pintsuk G and Thomser C 2012 Fusion Eng. Des. 871201 